\title{
BMJ Open Scoping review of the psychosocial aspects of infertility in developing countries: protocol
}

\author{
Mariam Salie (D) , ${ }^{1}$ Rizwana Roomaney (D) , ${ }^{1}$ Michelle Andipatin, ${ }^{2}$ Cal Volks ${ }^{3}$
}

To cite: Salie M, Roomaney $R$, Andipatin $\mathrm{M}$, et al. Scoping review of the psychosocial aspects of infertility in developing countries: protocol. BMJ Open 2021;11:e044003. doi:10.1136/ bmjopen-2020-044003

- Prepublication history and additional supplemental material for this paper are available online. To view these files, please visit the journal online (http://dx.doi.org/10.1136/ bmjopen-2020-044003).

Received 24 August 2020 Accepted 07 May 2021

\section{ABSTRACT}

Introduction Infertility is a widespread social phenomenon. For both women and men, there are several psychosocial issues associated with infertility. Numerous systematic reviews have focused on individuals' psychosocial issues pertaining to infertility, however, these have been conducted in developed countries. Thus, there is a gap in the current literature to analyse individuals' psychosocial issues associated with infertility in developing countries. Given that there are various contextual factors to consider for appropriate interventions to be implemented, it is vital to explore this topic in a developing context.

Methods and analysis A scoping review will be conducted. A total of 12 databases were identified and a search string including terms related to infertility, psychosocial aspects and developing countries was developed. The inclusion and exclusion of each article will be determined through the guidelines provided by the Preferred Reporting Items for Systematic Reviews and Meta-Analyses flow chart. Quantitative and qualitative data will be collated. The findings will summarise existing research on psychosocial aspects of infertility in developing countries and identify gaps in the research corpus.

Ethics and dissemination Data will not be collected from participants. Instead, data will be extracted from published studies and therefore no ethical approval is required. The findings will be published in a peer-reviewed journal.

\section{INTRODUCTION}

There are two types of infertility: primary and secondary. Primary infertility refers to the inability to achieve a clinical pregnancy after 12 months of regular unprotected sexual intercourse. ${ }^{1} \quad$ Secondary infertility refers to infertility that occurs after a prior successful conception, whether the conception resulted in a miscarriage, stillbirth or successful birth. ${ }^{1}$ Psychosocial aspects refer to the cognitive, emotional and social aspects of both the individual and the couple within their society. Several systematic reviews have been conducted in the field of psychosocial aspects of infertility. These existing reviews have evaluated the impact of infertility on marital and sexual relationships, quality of

\section{Strengths and limitations of this study}

This will be the first scoping review of psychosocial aspects of infertility in developing countries.

- The search strategy is extensive and includes 12 electronic databases with peer-reviewed articles, published conference proceedings, theses and dissertations.

- Researchers will also search bibliographies, unpublished conference proceedings, grey literature and unpublished theses and dissertations for inclusion.

- The key limitation in this review is the inclusion of only English literature.

- As this study is a scoping review, it will provide an overview of the literature and not necessarily address a specific research question.

life and psychosocial well-being of couples; ${ }^{2}$ the effect of psychosocial interventions on pregnancy rates, mental health and marital function among couples undergoing in vitro fertilisation (IVR); ${ }^{3}$ psychosocial interventions for infertile couples ${ }^{4}$ and individuals. ${ }^{5}$

Women who experience infertility may experience devastating social, psychological and economic consequences in developing countries. For example, in Africa, marriage without children is considered incomplete and insecure. Divorce or polygamy can follow. ${ }^{6-8}$ Umezulike and Efetie ${ }^{9}$ investigated 200 women with infertility in Nigeria. They found that $40 \%$ of male partners reported that they had extra marital affairs, 37\% reported that they had taken another wife and $12 \%$ of husbands stated that they were planning to divorce their wives. Leonard ${ }^{10}$ found women in Chad who had not been able to bear children risked being excluded from inheriting land and being able to make a living off the land. Africa carries a high burden of infertility disease, which ranges from $10 \%$ to $30 \% .^{6}$ Treatment for infertility is relatively neglected and is overshadowed by other illnesses. ${ }^{11}$ In addition, Dyer et $a l^{12}$ found that one in five couples (22\%) attending a state subsidised IVF clinic in the Western Cape in 
South Africa incurred catastrophic expenditure for infertility treatment. This expenditure was greater than $40 \%$ of annual expenditure (excluding food). Despite these factors, there is a dearth of research on psychosocial aspects of infertility conducted in developing countries.

Inhorn ${ }^{13}$ has detailed the role globally of medical anthropology's contribution to human reproduction, including the interaction of fertility with aspects of social life such as religion, cosmology, gender relations, marriage and divorce patterns, kinship, inheritance and more. Medical anthropology has made a contribution to understanding the lengths and practices people go to when faced with infertility. The field has demonstrated that infertility can lead to physical and emotional abuse and economic and social exclusion. ${ }^{14}$ Effective infertility treatments are less accessible in developing nations, where access to infertility treatment reflects class and race-based inequalities. ${ }^{15}$ Understanding the extent of research conducted among people living with infertility in the developing world using a psychological lens may assist in summarising the issues in a manner that gives expression to the psychological needs of these populations. This is an important step in developing psychological responses to these needs.

Previous review studies ${ }^{2-5}$ have focused on studies conducted in developed countries. The reviews demonstrate that research into infertility is increasing but that the vast majority of reviews cite studies conducted in developed countries. The binary concepts of developing/developed countries/economies are hotly contested constructs and depend on the classificatory systems used. ${ }^{16}$ The International Monetary Fund (IMF), the World Bank and the United Nations (UN) all have slightly different markers to categorise countries/economies. For the purpose of our study, we will use the World Economic Situation and Prospect's conceptualisation of developing countries/ economies which draws on data from multiple sources including the IMF, World Bank and UN committees. ${ }^{17}$ Neoclassical growth theories argued for homogeneity within countries/economies, however in contemporary societies, we see the heterogeneous nature of developing countries/economies. ${ }^{18}$ Vollrath $^{19}$ argues that a significant characteristic of developing economies/countries is the simultaneous existence of both a modern industrial economy and a more subsistence based economy. Thus, our conceptualisation of developing countries/economies takes into account the dual nature of economies within these regions.

While being cognisant of, and wanting to avoid binary conceptualisations of developed/developing economies, our literature search highlights the gaps in published knowledge from 'developing' regions/economies. Given this gap in knowledge, it is our contention that a scoping review utilising a psychological lens will provide robust data that will give form to these unexpressed psychological needs. A review of this nature will assist in understanding the scope of the problem so that meaningful, evidenced-based interventions can be developed in less advantaged countries. In addition, such a review contributes to the UN Sustainable Development goals (SDG's 4 \& 5) and consequently furthers a Social Justice Agenda.

\section{METHODS AND ANALYSIS}

Considering that no reviews have been conducted in this field, a scoping review seems the most appropriate. We will use the scoping review guidelines as explained by Arksey and O' Malley. ${ }^{20}$ Scoping reviews allow researchers to map broad areas investigated in a research area, and are useful for unexplored topics as they provide a framework to collate all the available literature on the topic. A highlight of the scoping review is the broad coverage and the freedom to include different study designs. ${ }^{20}$ This scoping review will allow us to explore published and unpublished research on psychosocial aspects of infertility in developing countries in order to identify themes and gaps.

\section{Stage one: identifying the research question}

The research team consulted to formulate the research question, which is as follows. 'What are the psychosocial aspects of infertility in developing countries?' In order to address this question, we seek to understand the psychosocial needs and experiences of individuals and couples with infertility, explore psychosocial counselling/interventions offered to individuals and couples with infertility in developing countries, and to identify the psychosocial support reported by individuals and couples with infertility in developing countries. These domains are not exclusive, as other emerging domains will be included.

\section{Stage two: identifying relevant studies}

A rigorous process was undertaken to ensure that the most relevant search databases are selected in the review. The research team consists of researchers based at three universities, each having access to several search databases. While there is considerable overlap in the databases that each team member has access to, there are some databases that team members are unable to access.

The first step in determining the databases to use in the scoping review involved each team member to list the databases that they are able to access. These lists of databases were then combined and duplicate databases were removed. Each researcher indicated the databases that they deemed most relevant (ie, databases that were in line with the aim of the scoping review). Team members did this by voting for these databases on a google sheet. We consulted with a subject librarian in making the final decision of the databases to be included. The databases to be included are as follows: Academic Search Premier-EBSCOhost, African digital repository-Sabinet, Elsevier, Clinical Key, Cochrane library, Google Scholar, PsycArticles, PsycInfo, Pubmed, Scopus, Web of Science, Proquest database of Dissertations and Theses. 


\section{Selection of keywords}

The research team worked with two subject librarians in developing a search string that will be used in the scoping review. The following search strategy was developed from a total of 21 terms and is based on three main concepts:

Concept 1: terms related to infertility-Infertility OR Involuntary childlessness OR Assisted reproduction OR ART OR Medically Assisted Reproduction OR MAR OR Secondary infertility.

AND concept 2: terms related to psychosocial aspects-culture OR religion OR spiritual* OR religious OR stigma OR psychosocial needs OR counselling OR family OR psychosocial impact OR maternal needs OR paternal needs OR tradition OR depress* OR anxiety OR Psychosocial Support Systems [mesh]).

AND concept 3: developing countries OR (name of country).

This search string will be used in each database to source articles for the review.

\section{Stage three: study selection}

The selection criteria was discussed and agreed on by all members of the team. Two members of the research team will independently conduct the literature search by applying the search string to the relevant databases and hand-searching grey literature. The team will first assess the titles and abstracts of articles to determine the articles that will be included in the review. Articles meeting the inclusion criteria will be retained and items that do not meet the inclusion criteria will be omitted from the review. Duplicate articles will be removed. Reviewers will independently categorise and list each article as either to be 'include', 'excluded' or 'uncertain'. The two independent reviewers will then compare their lists to determine which articles will be included. Any discrepancies will be assessed by a third member of the team. In cases where further clarification is required to include or exclude a study, the full text will be screened. The expected time period for the review is January-December 2021. The following inclusion and exclusion criteria have been identified.

\section{Inclusion criteria}

Publication data and type of article

All multidisciplinary studies published in English between 2000 and 2020; both primary and secondary studies. Unpublished conference proceedings as well as grey literature such as unpublished theses and dissertations will additionally be included. The authors will make contact with presenters and authors in this regard to request access.

\section{Patient and public involvement}

There will be no patient and public involvement in the study.

\section{Types of participants}

Males and females diagnosed with infertility. Data should have been collected in developing countries. These are

\section{Table 1 Data extraction form}

\section{Article reference}

\section{Country}

Paradigm

Aims and objectives

Participant characteristics

Data collection tools

Sample size

\section{Analysis}

Findings

1. General

2. Psychosocial needs

3. Emotional well-being and mental health

4. Support

5. Other

\section{Contribution of article}

countries identified as per the IMF conceptualisation of 'developing' countries (see Appendix A). Studies must include primary or secondary infertility. Studies can be qualitative (eg, exploratory) or quantitative (intervention, description, etc.).

\section{Article types}

All articles published in peer-reviewed journals, published protocols and grey literature-theses and dissertations. In addition, reference lists of included studies will be hand sorted for relevant articles.

\section{Exclusion criteria}

Studies in any language other than English, as well as theoretical papers.

\section{Stage four: data collection}

After selecting relevant studies for inclusion in our scoping review, we will chart the data. We will extract the relevant information from each study using a data charting form to guide the process (please refer to table 1 for the data charting form). Rayyan, a systematic review application, will be used to help us manage the articles and synthesise the extracted data. ${ }^{21}$ To begin with, the two reviewers will first independently chart the same five studies using the data charting form, as recommended by Levac et $a .^{22}$ They will then meet and compare the consistency of data extraction using the form. At this point, we will assess whether the research chart adequately allows us to answer our research question and purpose. The data extraction form will be revised, if needed. When consistency in data extraction has been achieved, we will continue the process of charting the data.

\section{Stage five: collating, summarising and reporting the results}

A scoping review provides a broad synopsis of all the material consulted. ${ }^{20}$ Quantitative data relating to type of study, setting and methodologies will be presented as frequencies in tables. Qualitative data will be summarised. 
Themes will be produced that will allow readers to gauge the breadth of psychosocial research on infertility that has been conducted in developing countries. In these themes, we will describe the psychosocial needs and experiences of participants, types of counselling and interventions offered to them and sources of support that were reported. Additional themes will also be reported on and gaps in the field of research will be identified.

\section{ETHICS AND DISSEMINATION}

No data will be collected from participants. Instead, data will be extracted from published studies available online. This research is therefore exempt from ethical approval.

The findings of this study will be published in a peerreviewed journal. We will seek to publish the findings either in journals that report on infertility or general health psychology journals. The findings will be of interest to researchers who intend on exploring psychosocial aspects of infertility among men and women in developing countries as it may provide a summary of research already conducted in this field and gaps for future research.

\section{Twitter Mariam Salie @MMacademic}

Contributors All authors contributed equally to the conceptualisation of the review and the writing of the protocol. MS, MA, RR and CV decided on the area of research. All authors were involved in designing the methodology of the scoping review. All authors contributed to the writing of the manuscript. MS was responsible for final formatting, editing and submission.

Funding The authors have not declared a specific grant for this research from any funding agency in the public, commercial or not-for-profit sectors.

Competing interests None declared.

Patient consent for publication Not required.

Provenance and peer review Not commissioned; externally peer reviewed.

Supplemental material This content has been supplied by the author(s). It has not been vetted by BMJ Publishing Group Limited (BMJ) and may not have been peer-reviewed. Any opinions or recommendations discussed are solely those of the author(s) and are not endorsed by BMJ. BMJ disclaims all liability and responsibility arising from any reliance placed on the content. Where the content includes any translated material, BMJ does not warrant the accuracy and reliability of the translations (including but not limited to local regulations, clinical guidelines, terminology, drug names and drug dosages), and is not responsible for any error and/or omissions arising from translation and adaptation or otherwise.

Open access This is an open access article distributed in accordance with the Creative Commons Attribution Non Commercial (CC BY-NC 4.0) license, which permits others to distribute, remix, adapt, build upon this work non-commercially, and license their derivative works on different terms, provided the original work is properly cited, appropriate credit is given, any changes made indicated, and the use is non-commercial. See: http://creativecommons.org/licenses/by-nc/4.0/.

\section{ORCID iDs}

Mariam Salie http://orcid.org/0000-0003-4060-4813

Rizwana Roomaney http://orcid.org/0000-0002-3585-4992

\section{REFERENCES}

1 World Health Organisation. Sexual and reproductive health, 2019. Available: https://www.who.int/reproductivehealth/topics/infertility/ definitions/en/

2 Luk BH-K, Loke AY. The impact of infertility on the psychological well-being, marital relationships, sexual relationships, and quality of life of couples: a systematic review. J Sex Marital Ther 2015;41:610-25.

3 Ying L, Wu LH, Loke AY. The effects of psychosocial interventions on the mental health, pregnancy rates, and marital function of infertile couples undergoing in vitro fertilization: a systematic review. J Assist Reprod Genet 2016;33:689-701.

4 Chow K-M, Cheung M-C, Cheung IK. Psychosocial interventions for infertile couples: a critical review. J Clin Nurs 2016;25:2101-13.

5 Boivin J. A review of psychosocial interventions in infertility. Soc Sci Med 2003;57:2325-41.

6 Dyer SJ. The value of children in African countries: insights from studies on infertility. J Psychosom Obstet Gynaecol 2007;28:69-77.

7 Dyer SJ, Abrahams N, Mokoena NE, et al. Psychological distress among women suffering from couple infertility in South Africa: a quantitative assessment. Hum Reprod 2005;20:1938-43.

8 Dyer SJ, Abrahams N, Hoffman M, et al. 'Men leave me as I cannot have children': women's experiences with involuntary childlessness. Hum Reprod 2002;17:1663-8.

9 Umezulike AC, Efetie ER. The psychological trauma of infertility in Nigeria. Int J Gynaecol Obstet 2004;84:178-80.

10 Leonard L. "Looking for children": the search for fertility among the Sara of southern Chad. Med Anthropol 2002;21:79-112.

11 van BalenF, Gerrits T. Quality of infertility care in poor-resource areas and the introduction of new reproductive technologies. Hum Reprod 2001;16:215-9.

12 Dyer SJ, Vinoos L, Ataguba JE. Poor recovery of households from out-of-pocket payment for assisted reproductive technology. Hum Reprod 2017;32:2431-6.

13 Inhorn MC. Quest for conception: gender, infertility and Egyptian medical traditions. Philadelphia, PA: University of Pennsylvania Press, 1994.

14 Inhorn MC. Right to assisted reproductive technology: overcoming infertility in low-resource countries. Int J Gynaecol Obstet 2009;106:172-4.

15 Inhorn MC, Birenbaum-Carmeli D. Assisted reproductive technologies and culture change. Annu Rev Anthropol 2008;37:177-96.

16 Nielsen L. Classifications of countries based on their level of development: How it is done and how it could be done. IMF working paper JEL Classification Numbers: O10, O15, O19, 2011. Available: https://www.imf.org/external/pubs/ft/wp/2011/wp1131.pdf

17 United Nations. Global economic monitoring unit (GEM). Available: https://www.un.org>document_gem>global-economic-monitoringunit

18 Temple J. Dual economy models: a primer for growth economists. The Manchester School 2005;73:435-78.

19 Vollrath D. The dual economy in long-run development. J Popul Econ 2009;14:287-312.

20 Arksey H, O'Malley L. Scoping studies: towards a methodological framework. Int J Soc Res Methodol 2005;8:19-32.

21 Rayyan. Rayyan QCRI. Available: http://rayyan.qcri.org

22 Levac D, Colquhoun H, O'Brien KK. Scoping studies: advancing the methodology. Implement Sci 2010;5:69. 